\title{
L'absolu et la dévastation des pommes de terres, ou le romantisme en question en 1837 (Lettres d'un voyageur, $\mathrm{X}$ )
}

Pierre Laforgue

\section{CpenEdition}

\section{Journals}

Édition électronique

URL : http://journals.openedition.org/recherchestravaux/191

DOI : 10.4000/recherchestravaux.191

ISSN : 1969-6434

Éditeur

UGA Éditions/Université Grenoble Alpes

Édition imprimée

Date de publication : 15 avril 2007

Pagination : 121-130

ISBN : 978-2-84310-107-7

ISSN : 0151-1874

Référence électronique

Pierre Laforgue, "L'absolu et la dévastation des pommes de terres, ou le romantisme en question en 1837 (Lettres d'un voyageur, X) », Recherches \& Travaux [En ligne], 70 | 2007, mis en ligne le 02 décembre 2008, consulté le 08 septembre 2020. URL : http://journals.openedition.org/ recherchestravaux/191; DOI : https://doi.org/10.4000/recherchestravaux.191 
Pierre LAFORGUE

Université de Franche-Comté

\section{L'absolu et la dévastation des pommes de terres, ou le romantisme en question en I837 (Lettres d'un voyageur, $\mathrm{X}$ )}

En cette année I837 où sont réunies les Lettres d’un voyageur paraissent également deux autres œuvres témoignant d'une pareille attitude critique à l'égard du romantisme, à savoir la première partie d'Illusions perdues de Balzac et les Lettres de Dupuis et Cotonet de Musset. Ensemble, ces trois ouvrages participent à une même remise en question du romantisme historique de I830, celui d'Hernani et de la Symphonie fantastique, pour prendre l'exemple de deux productions emblématiques de cette année phare, et chacun à leur façon Sand, Balzac et Musset montrent les limites de ce romantisme qui est aujourd'hui en train de vieillir dans les clichés et les stéréotypes. Les modalités auxquelles ils recourent pour montrer ces limites sont très différentes entre elles. Balzac procède historiquement, archéologiquement même, en remontant en 1820 à une sorte de protoromantisme, qui est en fait un paléoromantisme ${ }^{\mathrm{I}}$. Musset, lui, cultive la satire la plus mordante, en faisant dénoncer les poncifs du romantisme par deux habitants de la Ferté-sousJouarre à qui il fait jouer spirituellement le rôle de deux faux naïfs, avec comme résultat la représentation d'un romantisme caricatural. Sand, pour sa part, quel point de vue critique adopte-t-elle? Elle le dit en plusieurs passages

I. Sur ce sujet, nous nous permettons de renvoyer à nos deux articles : «Balzac, Chénier et le romantisme en I837, ou poésie et poétique dans Illusions perdues ", Cahiers Roucher-André Chénier, $\mathrm{n}^{\circ}$ 20, 200I, p. I27-I34 ; "Le débat romantique dans Illusions perdues, ou d'un romantisme l'autre ", Balzac, Illusions perdues, Paris, Presses de l'université de ParisSorbonne, 2003, p. 175-186. 
de son livre, et de façon parfaitement claire et explicite, son point de vue est celui de la fantaisie. Point de vue mais plus encore, en réalité, attitude devant la vie et les choses où sont engagées une philosophie de l'existence aussi bien qu'une poétique et une esthétique de la création littéraire.

La fantaisie dans les Lettres d'un voyageur est le premier élément d'un paradigme, dont les autres termes sont la Bohême, le bohémianisme, le vagabondage, mais aussi le divers, le bariolé (" prends tes voiles bariolées, ô ma chère fantaisie ! "; lettre X; p. 882 ; p. 260²). Ce paradigme de la fantaisie, qui est décliné à travers l'ouvrage avec autant de rigueur et de méthode que de liberté et d'improvisation', ne nous occupera pas ici en lui-même, et nous nous contenterons de poser qu'il est au cœur de l'entreprise de critique et de refondation du romantisme à laquelle se livre Sand dans les Lettres d'un voyageur. Ce qui nous intéressera, c'est de voir comment Sand engage dans l'œuvre un débat, quelquefois contradictoire, entre elle-même et le romantisme. Il ne s'agit pas, précisons-le, de ne pas tenir compte de la poétique et de l'esthétique de la fantaisie, mais simplement d'interroger, à l'intérieur même de cette poétique et de cette esthétique, la mise en œuvre de ce débat. Une telle étude devant requérir de longs et minutieux développements, nous restreindrons notre examen à quelques-uns des objets qui entrent dans ce débat romantique, comme, par exemple, l'usage de la rhétorique ou la pratique de l'ironie et du grotesque ; d'un autre côté, nous limiterons cet examen luimême à la seule lettre $\mathrm{X}$ des Lettres d'un voyageur, qui nous semble particulièrement exemplaire, et encore dans cette lettre seulement à une séquence de quelques pages. Cette lettre constitue le récit du voyage qu'effectue le Voyageur de la Vallée Noire à la vallée de Chamonix, pour rejoindre finalement Liszt et Marie d'Agoult. C'est une lettre joyeuse, et d'autant plus enjouée que Sand vient de gagner en dernière instance le procès qui l'opposait à son mari ; elle est en elle-même un bon exemple d'une écriture de la fantaisie ${ }^{4}$, et surtout, dans l'optique qui est la nôtre, par la contestation déli-

2. Selon la convention que nous adoptons pour ce volume: G. Sand, Lettres d'un voyageur, pages de l'édition de G. Lubin puis pages de l'édition de H. Bonnet.

3. Voir à ce propos l'excellente préface d'Henri Bonnet à son édition des Lettres d'un voyageur, op. cit., ainsi que nos deux études : «Fantaisie vénitienne ("Lettres d'un voyageur, II") ", Romanticoco. Fantaisie, chimère et mélancolie (I830-I860), Saint-Denis, Presses universitaires de Vincennes "L'Imaginaire du Texte ", 200I, p. I09-II8 ; « Les Lettres d'un voyageur, ou d'une écriture et d'une identité bohémiennes ", Corambé. Identité et fiction de soi chez George Sand, op. cit., p. 75-95.

4. Sur cette écriture de la fantaisie dans la lettre X, voir N. Mozet, George Sand, écrivain de romans, op. cit., p. 44-47. 
bérée des codes romantiques qui y est pratiquée avec méthode et bonne humeur, elle pose très nettement la question du romantisme, dans le sens où celui-ci est envisagé selon deux enjeux, l'un critique, l'autre problématique.

Avant d'essayer d'apprécier dans la lettre X cette double dimension critique et problématique, qui est à nos yeux le propre du romantisme de la fin des années 1830, et afin, d'autre part et conjointement, de montrer l'origine de cette attitude contestataire à l'égard du ci-devant romantisme de I830, nous nous livrerons, à la façon de Dupuis et de Cotonet, à un semblant d'inventaire, aussi rapide que sommaire, des procédés de l'écriture romantique, tels qu'ils sont en quelque sorte fixés à l'époque, dans des œuvres très représentatives comme Lélia, Le Lys dans la vallée ou les Nuits. Ce que ces différents textes peuvent avoir en commun c'est, en maints endroits, une exaltation oratoire et lyrique qui se traduit par le recours systématique à l'adjectif ainsi que par l'usage constant de l'apostrophe et de l'exclamation, comme si entre cette parole et le monde il n'y avait aucune distance, ou du moins qu'une distance extrêmement réduite. Il ne s'agit évidemment pas de soutenir absurdement que ces œuvres cèdent à un lyrisme irrépressible et irréfléchi : tout, au contraire, témoigne que de telles œuvres obéissent à un projet d'écriture parfaitement concerté et conscient de lui-même. Il n'est ici question que de constater que c'est dans une quasi-immédiateté d'elles-mêmes au monde que ces œuvres prennent sens, à des degrés divers et selon des modalités différentes : le dolorisme pélicanisé du poète mussétien n'est pas entièrement comparable au désespoir frénétique et frigide de Lélia ni non plus à la tristesse regrettante et mélancolique de Félix de Vandenesse.

Pas sur le même plan, mais dans le même ordre d'idées, on isolera un autre trait de l'écriture romantique de I830, la présence nombreuse, surabondante, des métaphores et des comparaisons, selon une fréquence telle que Pierre Leroux, dans son grand article du Globe sur les Orientales (8 avril I829), y a vu une des caractéristiques du "style romantique " (l'accumulation de métaphores aboutit à la création d'une nouvelle figure, le symbole). Comme les exclamations et les apostrophes, les comparaisons et les métaphores, dans l'abus même qui est fait d'elles, disent l'immédiateté de l'écriture à son objet. C'est très nettement visible dans la pratique romantique de la description et, plus profondément, du descriptif. Du joli descriptif, faudrait-il ajouter, qui relève de ce que nous appellerons le bien-écrire romantique en I830. Et de même qu'il n'est pas possible à l'époque de raconter une histoire sans pousser des cris, des plaintes ou des gémissements, de même on ne peut pas décrire sans recourir à des métaphores et à des comparaisons. Celles-ci sont non pas les moyens de la description, son support, mais carrément sa substance. Tout le système descriptif du Lys dans la vallée le montre admirablement, avec pour 
contrepartie, et spécialement dans ce roman, une inflation de poétismes qui concourent à un beau style - très fleuri.

Voilà très grossièrement présenté un état romantique des choses en I837, alors que ce mouvement littéraire est bien établi et se trouve en bonne voie d'institutionnalisation. Il est compréhensible, dans ces conditions, que se soit produite une réaction à l'encontre de ce qui risquait de devenir un nouvel académismes. Cette réaction, ce sont les écrivains romantiques eux-mêmes, et non pas leurs adversaires, comme Nisard, qui, à partir de leur propre horizon d'attente, l'ont mise en place et l'ont promue : ce qui explique, entre autres choses, que le Balzac du Lys dans la vallée écrive dès l'année qui suit cette œuvre hyperromantique la première partie d'Illusions perdues, ou que le Musset des Nuits soit l'ironiste des Lettres de Dupuis et Cotonet - et que l'auteur de Lélia soit aussi celui des Lettres d'un voyageur.

(Avant d'en venir aux Lettres d'un voyageur elles-mêmes, et en particulier à la lettre $\mathrm{X}$, nous ouvrirons rapidement une courte parenthèse pour insister sur le fait qu'il importe à cet égard de tenir compte de la date des différentes lettres, écrites entre I834 et I836, et de celle de leur réunion en I837, et pour noter qu'historiquement le romantisme flamboyant des deux premières lettres n'a pas grand-chose à voir avec celui du recueil, fantaisiste et distancié, trois ans plus tard. De là des effets de sens et de distorsion dans le sens qui ne doivent pas être négligés, puisqu'ils contribuent à la perspective textuelle singulière de l'œuvre, qui joue constamment du décentrage.)

Passons à la lettre $\mathrm{X}$, donc, et aux deux enjeux critique et problématique qu'elle illustre. Enjeu critique, tout d'abord, et c'est à travers la relation que Sand entretient dans cette lettre à la métaphore que nous l'envisagerons, puisque nous avons avancé que la métaphore est un des éléments constitutifs de l'écriture romantique. Nous nous occuperons pour cela d'une seule page, où, à travers une description, c'est tout le système rhétorique de la métaphore qui est mis en cause et dénoncé par Sand. Voici cette page :

Ce que je sais, c'est que cette ligne de feux, établis comme des signaux tout le long du ravin, m'offrit, au milieu de la nuit, un spectacle magnifique. Ils perçaient de taches rouges et de colonnes de fumée noire le rideau de vapeur d'argent où ma vallée était entièrement plongée et perdue. Au-dessus des feux, au-dessus de la fumée et de la brume, la chaîne du Mont-Blanc montrait une de ces dernières ceintures granitiques, noire comme l'encre et couronnée de neige.

5. Le danger était réel et, en I846 encore, Baudelaire, dans le Salon de cette année-là, dénonçait et stigmatisait l'académisme d'un certain romantisme, celui de Hugo au premier chef, pour promouvoir un autre romantisme, dont Delacroix aurait été le chef de file : voir Ch. Baudelaire, Salon de I846, in Euvres complètes, édition de Cl. Pichois, Paris, Gallimard "Bibliothèque de la Pléiade », t. II, I976, p. 43I. 
Ces plans fantastiques du tableau semblaient nager dans le vide. Sur quelques cimes que le vent avait balayées, apparaissaient, dans un firmament pur et froid, de larges étoiles. Ces pics de montagnes, élevant dans l'éther un horizon noir et resserré, faisaient paraître les astres étincelants. L'œil sanglant du Taureau, le farouche Aldébaran, s'élevaient au-dessus d'une sombre aiguille, qui semblait le soupirail du volcan d'où cette infernale étincelle venait de jaillir. Plus loin, Fomalhaut, étoile bleuâtre, pure et mélancolique, s'abaissait sur une cime blanche, et semblait une larme de compassion et de miséricorde tombée du ciel sur la pauvre vallée, mais prête à être saisie en chemin par l'esprit perfide des glaciers.

Ayant trouvé ces deux métaphores, dans un grand contentement de moi-même, je fermai ma fenêtre. Mais en cherchant mon lit, dont j'avais perdu la position dans les ténèbres, je me fis une bosse à la tête contre l'angle du mur. C'est ce qui me dégoûta de faire des métaphores tous les jours subséquents. Mes amis eurent l'obligeance de s'en déclarer singulièrement privés. (P. 902 ; p. 279-280.)

La description est somptueuse, c'est un nocturne neigeux sur fond d'étoiles. Le mont Blanc de nuit est saisi dans une paradoxale noirceur, laquelle, selon la figure organisatrice de l'inversion qui régit toute la poétique de la description dans cette page, est perçue comme principe de lumière. Au sens propre on est en présence d'une lumière de nuit dont la luminosité singulière est rehaussée par l'éclat des étoiles. Or, dans la perspective que nous avons adoptée, le plus intéressant ici est le traitement par Sand de la métaphore, ou plutôt du métaphorique. Toute la description, en effet, est portée par la métaphore et cet investissement du descriptif par le métaphorique contribue à faire de cette page un véritable morceau de bravoure, une sorte d'ekphrasis romantique, ou romantisant. Sand, la première, a conscience de cet envahissement des images, métaphores et comparaisons, et le dénonce avec esprit, sinon avec exactitude, tant il est clair que le recours à la métaphore ne se limite pas aux deux occurrences qu'elle signale. Elle n'en est pas elle-même avare dans les Lettres d'un voyageur, et c'est la figure la plus répandue qui soit dans le texte. Ce qui importe, ce n'est pas qu'elle use de métaphores ni que ces métaphores soient associées à la description, mais que soit instauré un soupçon critique à leur endroit, comme si, de temps à autre, pas tout le temps il est vrai, était contestée par l'écrivain romantique qu'elle est cette métaphorisation de la réalité à laquelle elle se livre. À travers cette contestation, ce n'est pas la rhétorique ni même la poétique de la métaphore qui sont attaquées facétieusement, mais proprement la métaphysique sur laquelle se fonde cette conception de l'écriture comme métaphore de la réalité : cette conception implique que la métaphorisation est le mode d'être de l'écriture au monde, en ce sens que le monde n'est susceptible d'être non seulement représenté, mais simplement appréhendé que par la métaphore. Avec pour première conséquence littéraire l'extravagance, la gratuité, bref la fantaisie, mais une fantaisie qui se complairait dans le propre exercice de son inventivité. 
Passons à l'enjeu problématique, maintenant, tel qu'il se dégage de cette lettre $\mathrm{X}$. Cet enjeu est problématique, en ce sens que se problématise dans cette lettre une conception du romantisme en rupture par rapport à l'ordinaire conception du romantisme de naguère. Cette ci-devant conception est de nature philosophique, elle postule que l'artiste, l'écrivain, en un mot le poète est investi d'une mission, qu'il exerce un sacerdoce, etc. et que sa fonction est chose sérieuse, religieuse même. Pour autrement dire, guide de l'humanité, il se doit d'annoncer aux hommes la bonne nouvelle de leur bonheur à venir et cet évangile humanitaire s'appuie sur un certain nombre de mythes dont les plus importants sont le Peuple, la Révolution, le Progrès, la Liberté. Toutes ces matières sont évoquées dans la lettre VI, mais c'est l'impétueux et énergique Éverard qui défend cette vision des choses; face à lui, «le petit George " (p. 785 ; p. I68) défend les droits de l'artiste à pratiquer une " école buissonnière » et à se comporter comme un "voyou " (p. 780 ; p. I64), idéologiquement doit-on comprendre, qui ne respecte pas les convenances politiques et n'a pas foi dans le credo philanthropique de gauche. Ce moineau franc se refuse donc à rien conclure (p. 785 ; p. I68).

Le même désengagement idéologico-politique se lit dans la lettre $\mathrm{X}$, si ce n'est que le débat s'y formule de manière beaucoup plus subtile que dans la lettre VI. Ce débat fait l'objet du centre de la lettre X qui est occupé par le récit du séjour en Suisse, où le Voyageur retrouve Liszt et Marie d'Agoult, et excursionne, de Genève à Fribourg, avec eux et en compagnie de l'inénarrable major Pictet. Ce dernier personnage est très féru de philosophie allemande et, à califourchon sur sa mule, au sein des paysages les plus pittoresques, ne voit rien des beautés qui l'entourent. Il passe son temps à argumenter avec Sand. La discussion entre elle et le major est de nature philosophique, ou du moins le major voudrait qu'elle le fût, alors que son interlocuteur, préférant admirer le paysage, ne cesse de tourner en dérision ses prétentions à la philosophie. De fait, il y a de quoi, tant le major parait obnubilé par une phrase aussi profonde qu'incompréhensible : "L'absolu est identique à lui-même" (p. 906 ; p. 283). Dans le meilleur des cas, c'est une tautologie, mais, en tout état de cause, il est absurde au milieu des beautés de la nature et alors que l'on se promène d'avoir l'esprit occupé par de semblables réflexions. C'est ce que Sand essaie de faire comprendre au major. En vain. Faut-il considérer dans ces conditions que c'est Sand, le Voyageur, qui a raison et le major qui a tort et que, contre la philosophie, le spectacle de la nature doit l'emporter? Ce n'est pas certain du tout, surtout si l'on tient compte de l'intéressant jugement qui est mis dans la bouche d'Arabella, alias Marie d'Agoult, qui met un terme à la discussion aigre-douce entre Sand et le major par ces mots : «Il y a quelque chose de plus stupide que l'indifférence du vul- 
gaire en présence des beautés naturelles ; c'est l'extase obligée, c'est l'infatigable exclamation " (p. 906 ; p. 283). Dans le contexte, c'est directement adressé au personnage du Voyageur, et assez clairement Arabella prend parti contre lui. Mais en réalité, Arabella n'existe que comme personnage de fiction, c'est une invention de Sand, même si "Arabella », avec des guillemets, désigne dans la "réalité ", et toujours avec des guillemets, Marie d'Agoult. D'autre part, et plus profondément, la réflexion prêtée à ce personnage d'Arabella n'est pas que de circonstance, elle va beaucoup plus loin qu'il ne semble et peut connaître une application à l'ensemble même des Lettres d'un voyageur. En cela, ces propos participent de la remise en question du romantisme que l'on voit à l'œuvre dans les Lettres d'un voyageur. Avec un sens très fin de l'autocritique Sand fait mettre, par le truchement du personnage d'Arabella, le doigt sur l'un des aspects les plus voyants de l'ouvrage, la multiplication des exclamations et des exclamatives dans les différentes lettres, lequel aspect est aussi, faut-il le dire, l'un des plus visibles du romantisme en général. Et l'on s'est assez moqué de cet enthousiasme des écrivains romantiques et de leurs thuriféraires; qu'il suffise de penser à $\mathrm{M}^{\mathrm{me}}$ de Bargeton dans la première partie d'Illusions perdues.

Contre ces exclamations irréfléchies, ce serait donc la réflexion philosophique qui serait finalement prônée. En un sens, mais en un certain sens seulement : le texte ne donne pas raison à l'un des deux protagonistes plutôt qu'à l'autre et l'épisode ne se termine pas sur ce jugement d'Arabella. La conclusion intervient quatre pages plus loin, lorsque, arrivée à Fribourg, la petite troupe se rend à l'église de Saint-Nicolas pour y admirer « le plus bel orgue qui ait été fait jusqu'ici » (p. 910 ; p. 287). C'est l'occasion d'une page ébouriffante de drôlerie. Est raconté pour l'occasion comment, Liszt s'étant mis à l'instrument, l'organiste peu à peu lui prend sa place et entreprend lui-même de jouer :

À vrai dire, celui-ci [Liszt] ne tirait pas tout le parti possible de la machine. Il cherchait platement les sons les plus purs et ne nous régalait pas du plus petit coup de tonnerre. Aussi l'organiste de la cathédrale, gros jeune homme à la joue vermeille, confrère familier et quasi protecteur de notre ami, le poussait doucement à chaque instant, et, prenant sans façon sa place, essayait à force de bras, de nous faire comprendre la puissance vraiment grande, je le confesse, du charlatanisme musical. Il fit tant des pieds et des mains, et du coude, et du poignet, et, je crois, des genoux (le tout de l'air le plus flegmatique et le plus bénévole), que nous eûmes un orage complet, pluie, vent, grêle, cris lointains, chiens en détresse, prière du voyageur, désastre dans le chalet, piaulement d'enfants épouvantés, clochettes de vaches perdues, fracas de la foudre, craquement des sapins, finale, dévastation des pommes de terre. (P. 9II ; p. 288.) 
À la façon de Liszt lui-même ${ }^{6}$, à moins qu'elle n'imite Beethoven donnant des titres aux différents mouvements de sa Symphonie pastorale, Sand consigne avec virtuosité le programme de ce poème symphonique pour orgue seul. À l'évidence, l'énumération cocasse des différents épisodes tragicomiques amenés par l'orage relève d'une esthétique du grotesque et nul doute que la magnifique et grandiose mention finale de la « dévastation des pommes de terre " ne constitue le sommet épique de ce crescendo farcesque. Comme si la dimension bouffonne n'était pas déjà assez grande, le Voyageur immédiatement après félicite l'organiste par les mots suivants :

Monsieur, cela est magnifique : je vous supplie de me faire encore entendre ce coup de tonnerre; mais je crois qu'en vous asseyant brusquement sur le clavier vous produiriez un effet plus complet encore. (P. $912 ;$ p. 288.)

Il sera toujours possible de supposer que de cette façon le malheureux cacophoniste pourra faire la preuve que "l'absolu est identique à lui-même ", mais nous préférerons considérer que ce concert improvisé fait pendant à la discussion que Sand et le major viennent d'avoir quelque temps auparavant, et qu'il lui fait pendant non pas d'un point de vue thématique, mais d'un point de vue poétique : au sérieux de l'objet de cette discussion (pas le sérieux de la discussion, puisqu'il n'y a nul sérieux au grand dam du major) correspondant la bouffonnerie de l'improvisation musicale. L'essentiel est d'imprimer aux choses une espèce de dérive grotesque qui vienne dynamiter la possibilité de tenir un discours, avec ce qu'un discours suppose de cohérence et de cohésion, d'organisation, de suivi, avec ce qu'un discours suppose aussi de transitivité de la pensée (ce qu'Éverard appelait dans la lettre VI « conclusion »; p. 785 ; p. 1687). L'épisode musical, si l'on peut dire, de Fribourg a dans cette optique pour fonction de ruiner ou plutôt d'achever de ruiner la possibilité d'un discours philosophique sérieux, en introduisant dans la narration une tonalité bouffonne qui est presque d'une " hénaurmité " préflaubertienne. Bien sûr, Sand a conscience que cette fonction dévolue à la musique n'est pas pleinement admissible et c'est pourquoi une lettre accordant à la musique la place qui lui revient véritablement s'insère aussitôt après dans l'ouvrage (la lettre XI à Meyerbeer) et fait office d'appendice de grand style. Cela n'empêche pas que la bouffonnerie de cet épisode de la lettre X est pleine de sens et que c'est très précisément cette bouffonnerie qui porte le sens lui-même.

6. Voir dans l'édition de G. Lubin, la note n ${ }^{\circ}$ 5, p. 9II, où il cite à titre d'exemple une note marginale de Liszt à l'une de ses compositions.

7. Voir aussi mon commentaire sur ce sujet dans Corambé. Identité et fiction de soi chez George Sand, op. cit., p. 87-88. 
Pour finir, on comprend bien qu'il ne s'agit pas d'opposer philosophie et musique (il n'y a ni philosophie ni musique), ni non plus de voir une antithèse entre l'absolu et la dévastation des pommes de terre (celui-là est défendu par qui l'on désignait au XVIII siècle du nom d'espèce et celle-ci est à mettre sur le compte d'un organiste à qui la musique est étrangère). Ce qui est en jeu, c'est une attitude fantaisiste de l'écrivain en face de l'être, où se conjuguent rieusement et sérieusement adhésion au monde et distance par rapport à lui, selon une poétique qui a été celle du romantisme pendant des années, mais qui connaît maintenant une inflexion.

En conclusion, nous vérifierons rapidement les analyses qui précèdent, en constatant que de l'édition originale de I837 à la deuxième édition de I843, la dénonciation du romantisme au moyen de la fantaisie s'est accentuée. L'emprise de plus en plus grande de la fantaisie n'a rien d'étonnant, si l'on considère qu'au fil des années le romantisme perd de plus en plus de la signification poétique et philosophique, politique aussi, qu'il avait autour de I830. Dès lors donc qu'il n'a plus sur la réalité la même prise idéologique, il est compréhensible que la distance littéraire soit encore plus grande à l'égard de l'écriture romantique et que cette mise à distance se traduise par une accentuation de la part dévolue à la fantaisie, celle-ci constituant désormais à elle seule toute la relation de l'écriture à la réalité. C'est ainsi que l'on observera dans cet ordre d'idées que Sand dans l'édition de I843 a systématiquement éliminé dans l'épisode suisse de la lettre $\mathrm{X}$ toutes les références à la philosophie politique des années I830, de Saint-Simon à Fourier en passant par Lamennais. Bien sûr, on peut avancer que c'est parce que maintenant elle est devenue la prosélyte de Leroux : mais on ne trouve pas la moindre référence à Leroux dans la deuxième édition des Lettres d'un voyageur. L'important, alors, était-il de déréférentialiser politiquement et idéologiquement le texte, afin de le faire basculer complètement du côté de la fantaisie ? Peut-être, à cette réserve près que dans la préface qui désormais précède le recueil un coup de barre politique est donné, où l'accent est mis sur le mal du siècle et les remèdes philosophiques, religieux et politiques que l'on y peut apporter. Sans porter un jugement de valeur en faveur de l'édition originale au détriment de la deuxième édition, nous nous bornerons à constater que la poétique de la fantaisie en I843 semble réclamer un dispositif textuel dont les Lettres en I837 se passaient avec une grâce magique, qui n'était que l'autre nom de la poésie. Peut-être aussi, et ce sera sur cette hypothèse que nous nous arrêterons, que l'introduction dans le monde de Sand de « l'errante et fugitive 
Consuelo ${ }^{8}$ " en I842-I843 a modifié le sens et la signification du voyage au pays des chimères et que la "verte Bohême " (lettre VI ; p. 8I7 ; p. I98), en devenant un espace du réel, s'est chargée d'un poids jusqu'alors inconnu, celui de l'histoire, et que, confrontée aux « révolutions formidables ${ }^{9}$ » sur lesquelles débouche le roman, la fantaisie déploie ses « voiles bariolées » (lettre $\mathrm{X}$ ; p. 882 ; p. 260) à d'autres vents.

8. G. Sand, Consuelo, chap. XXIII, édition de R. Bourgeois et S. Vierne, Meylan, Les Éditions de l'Aurore, I983, t. I, p. 196.

9. G. Sand, «Notice ", Consuelo, op. cit., t. I, p. 39. 
LES LETTRES ET LES ARTS 